\title{
Cardiovascular Comorbidities in Psoriasis and Psoriatic Arthritis: Pathogenesis, Consequences for Patient Management, and Future Research Agenda: A Report from the GRAPPA 2009 Annual Meeting
}

\author{
WOLF-HENNING BOEHNCKE, DAFNA D. GLADMAN, and VINOD CHANDRAN
}

\begin{abstract}
Psoriasis is often associated with other diseases, substantially adding to the patient's burden of disease. Recent epidemiologic studies have demonstrated an increased cardiovascular morbidity among patients with psoriasis and psoriatic arthritis (PsA), which contributes to their reduced life expectancy. At the meeting of the Group for Research and Assessment of Psoriasis and Psoriatic Arthritis (GRAPPA) adjacent to the International Federation of Psoriasis Associations (IFPA) congress, members discussed the pathogenetic aspects of this association and resulting consequences for the management of patients with psoriasis and PsA. A future research agenda was considered. (J Rheumatol 2011;38:567-71; doi:10.3899/jrheum.101124)
\end{abstract}

Key Indexing Terms:

PSORIATIC ARTHRITIS PSORIASIS

MYOCARDIAL INFARCTION
INSULIN RESISTANCE ATHEROSCLEROSIS ANTI-TUMOR NECROSIS FACTOR AGENTS
Given the central role of the immune system in pathogenesis $^{1,2,3,4}$, psoriasis and psoriatic arthritis (PsA) are currently considered to be immune-mediated inflammatory disorders (IMID), alongside numerous others such as rheumatoid arthritis (RA) $)^{5}$. Despite their distinct clinical presentation, these diseases share many common features such as their chronic course, inflammatory expression, and several pathogenetic aspects, including the central role of the immune system; a Th1-like cytokine milieu in the affected tissue, dominated by interferon- $\gamma$ and interleukins 2 and 22; and the key role of tumor necrosis factor- $\alpha$ (TNF- $\alpha$ ). The profound therapeutic effect of strategies to neutralize TNF- $\alpha$ have revolutionized the management of RA and have begun to alter the way psoriasis and PsA are being treated ${ }^{6,7}$.

Another shared feature of IMID is their association with other diseases, namely cardiovascular diseases (CVD) such as atherosclerosis and myocardial infarction. It is these comorbidities that have a direct impact on the mortality associated with IMID $^{8}$. The exact mechanism by which

From the Department of Dermatology, Johann Wolfgang Goethe University, Frankfurt am Main, Germany; and Division of Rheumatology, Department of Medicine, University of Toronto, Centre for Prognosis Studies in the Rheumatic Diseases, Toronto Western Hospital, Toronto, Ontario, Canada.

W.H. Boehncke, MD, Department of Dermatology, Johann Wolfgang Goethe University; D.D. Gladman, MD, FRCPC, Professor of Medicine; $V$. Chandran, MBBS, MD, DM, Clinical Fellow, Division of

Rheumatology, Department of Medicine, University of Toronto, Centre for Prognosis Studies in the Rheumatic Diseases, Toronto Western Hospital.

Address correspondence to Dr. W.H. Boehncke, Department of

Dermatology, Johann Wolfgang Goethe-University,

Theodor-Stern-Kai 7, D-60590 Frankfurt am Main, Germany.

E-mail: boehncke@em.uni-frankfurt.de these diseases predispose a patient to CVD is unclear, but may involve various cellular and humoral inflammatory mediators, namely TNF- $\alpha^{9}$. Recently, circulating concentrations of TNF receptors were shown to be highly predictive of mortality in patients with $\mathrm{RA}^{10}$, and treating RA with TNF- $\alpha$ blockers substantially reduced the cardiovascular morbidity and mortality in responding patients ${ }^{11}$.

Clinical aspects of cardiovascular comorbidity in psoriasis. In a landmark report ${ }^{12}$ describing the findings in a cohort of almost 3000 patients, Henseler and Christophers discussed the association of psoriasis with diabetes mellitus, obesity, heart failure, and hypertension. Their observations have since been reproduced in multiple studies. Cardiovascular morbidity has a substantial influence on patients' life expectancies, which are 3.5 years shorter in men and 4.4 years shorter in women with severe psoriasis ${ }^{13}$. Mallbris, et al were able to establish an association between psoriasis severity and cardiovascular mortality ${ }^{14}$, which was confirmed by Gelfand, et al, who reported up to 3-fold increased risk for psoriasis patients to develop myocardial infarction, depending on age and disease severity ${ }^{15}$. In a carefully performed case-control study, Ludwig, et al observed coronary artery calcification to be much more frequent and pronounced in patients with psoriasis compared to controls matched for all known risk factors ${ }^{16}$. As coronary artery calcification reflects coronary artery disease, these results further substantiate the research of Mallbris and Gelfand.

Clinical aspects of cardiovascular comorbidity in PsA. Although large-scale epidemiologic studies in PsA are not Personal non-commercial use only. The Journal of Rheumatology Copyright @ 2011 . All rights reserved. 
available, clinic-based studies have shown that PsA is associated with increased mortality risk ${ }^{17,18}$. CVD were the primary cause of death for a large proportion of the cases in this prospective study ${ }^{18}$, and the standardized prevalence ratios for myocardial infarction, angina, and hypertension were reported to be significantly higher in patients with PsA than the general population ${ }^{19}$. Factors associated with CVD included diabetes, hyperlipidemia, and high Psoriasis Area and Severity Index (PASI) scores ${ }^{19}$. Thus, severe psoriasis is an important predictor for CVD in patients with PsA.

PsA is associated with subclinical atherosclerosis. The association of hyperuricemia with hypercholesterolemia and renal impairment in PsA was reported in $2000^{20}$. A subsequent study in PsA patients without clinically evident CVD showed that there is a correlation between serum uric acid concentration and subclinical atherosclerosis as measured by carotid intima-media wall thickness (IMT) ${ }^{21}$. Hyperuricemia is an independent risk factor for CVD and may be a marker of subclinical atherosclerosis in $\mathrm{PsA}^{22}$. Tam, et al found that subclinical atherosclerosis, defined using carotid IMT, was increased in PsA patients compared to matched controls $^{23}$. Increased blood sugar and total triglyceride levels were independently associated with subclinical atherosclerosis in PsA. Of interest, the Framingham risk score was similar in PsA patients with and without subclinical atherosclerosis; $35 \%$ of the patients had subclinical atherosclerosis despite having a low cardiovascular risk score ${ }^{23}$. Similar results were obtained by Eder, et al from Israel ${ }^{24}$ and Gonzalez-Juanatey, et al from Spain ${ }^{25}$.

An additional factor in the predisposition to CVD among patients with PsA is the use of nonsteroidal antiinflammatory drugs (NSAID). Both Cox-2-selective and nonelective NSAID have been associated with an increased risk for CVD $^{26}$. Because many patients with PsA use NSAID, their risk for cardiovascular complications may be increased.

The effect of anti-TNF therapy with onercept on traditional and novel biochemical cardiovascular risk factors was evaluated at baseline and at the end of the treatment in a double-blind, placebo-controlled study in 127 patients with $\mathrm{PsA}^{27}$. Onercept $100 \mathrm{mg}$ induced significant reductions in concentrations of C-reactive protein, lipoprotein(a), and homocysteine, and an increase in the serum sex hormone-binding globulin, apolipoprotein (Apo) AI, Apo B, and triglycerides. These results suggested that it was not possible to prove that TNF blockade would provide cardioprotection on the basis of biochemical changes in isolation, and direct measures of atherosclerotic progression, such as carotid ultrasound, may be better ${ }^{27}$. In a pilot study, Tam, et al showed that short-term TNF- $\alpha$ blockade may be associated with reduction of IMT in PsA patients, along with improvement in clinical and laboratory indices of inflammation, but independent of changes in lipid profiles ${ }^{28}$. Anti-TNF- $\alpha$ therapy was also shown to improve aortic stiffness in patients with inflammatory arthropathies, including PsA ${ }^{29}$. These findings support the favorable effect of anti-TNF treatment on cardiovascular risk in patients with PsA.

Pathogenetic aspects of cardiovascular comorbidity in psoriasis and PSA. Pathophysiologically, the increased cardiovascular mortality of psoriasis patients is a consequence of what has been termed the "psoriatic march" (Figure 1) ${ }^{30}$. Psoriasis and its comorbidities, primarily obesity, contribute to the inflammatory burden of the affected patient. Systemic inflammation in turn causes insulin resistance, where the equilibrium between pro- and antiatherogenic effects of insulin is shifted toward the former (Figure 2) ${ }^{31}$. Insulin resistance results in endothelial dysfunction, which in turn leads to atherosclerosis and subsequent myocardial infarction, if coronary arteries are involved ${ }^{32}$.

\section{Clinical consequences}

Lifestyle interventions. The data reviewed here suggest a substantially elevated risk for CVD in patients with psoriasis and PsA. As psoriasis and PsA can only be controlled and not cured, it is of utmost importance for these patients to eliminate any additional risk factors for CVD that they can influence directly, particularly obesity and smoking. As both are prevalent among psoriasis patients ${ }^{33}$, clinicians need to convince their patients to normalize body weight and to quit smoking. Lifestyle interventions such as those practiced in the management of diabetes mellitus should be recommended.

Considerations regarding systemic therapies. When considering treatment options for psoriasis, it is evident that sever-

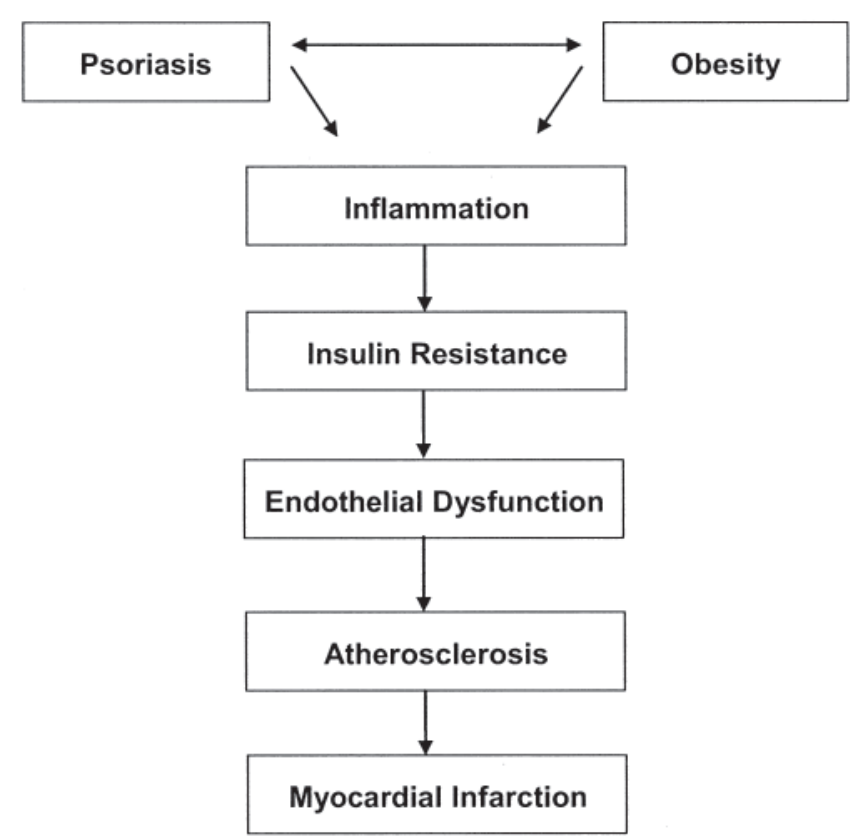

Figure 1. The "psoriatic march." Psoriasis and its comorbidities, primarily obesity, contribute to the inflammatory burden of the affected patient. Systemic inflammation in turn causes insulin resistance. This results in endothelial dysfunction, which provides the basis for atherosclerosis and subsequently myocardial infarction, if coronary arteries are involved. 


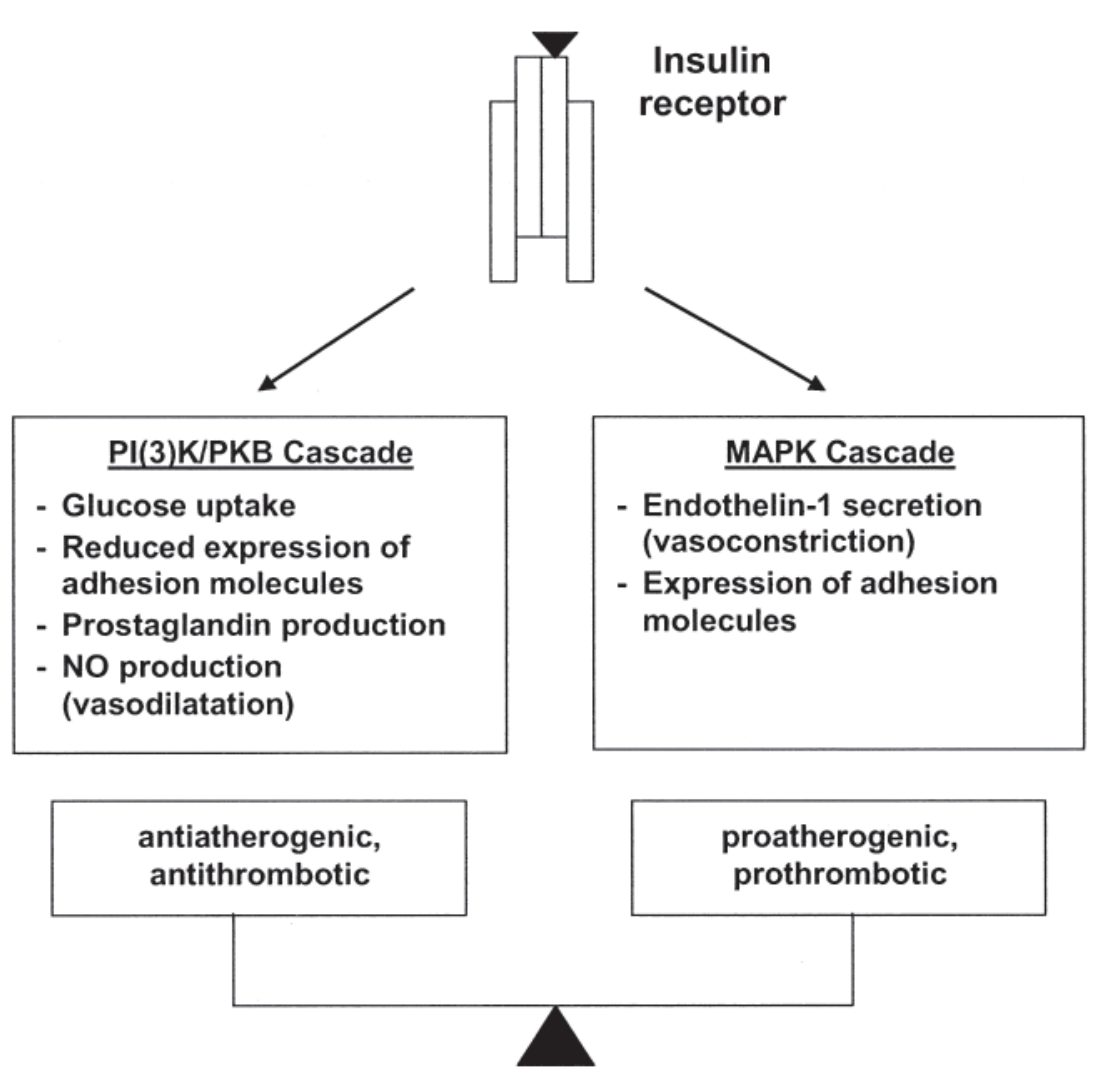

Figure 2. Insulin resistance as a cause for endothelial cell dysfunction. Physiologically, endothelial cells respond to insulin by producing vasodilating nitric oxide (NO) via the endothelial nitric oxide synthase (eNOS) pathway (left). With insulin resistance, this pathway is blunted, but insulin's mitogenic actions, which are mediated via the mitogen-activated protein kinase (MAPK) pathway, remain intact, potentially leading to increased production of adhesion molecules, thereby predisposing insulin-resistant patients to hypertension and atherosclerosis (right) (adapted from Boehncke, et al. Br J Rheumatol 2007;157:1249-51 ${ }^{31}$ ); with permission.

al systemic antipsoriatic therapies may cause or worsen cardiovascular comorbidity. This is particularly true for acitretin, which may increase serum triglycerides and cholesterol, and cyclosporin A, which may cause difficult-to-control hypertension. Side effects must be taken into account, and appropriate monitoring must be conducted to minimize these additional risks ${ }^{34}$.

Comorbidity necessitates comedication. In a survey of 1200 psoriasis patients, Mrowietz and coworkers found that only one-third took no other comedications, whereas one-quarter took more than 3 other systemic therapies ${ }^{35}$. Many of these concomitant medications are known to trigger psoriasis; this has been well established for beta-blockers and angiotensin-converting enzyme (ACE) inhibitors (Table 1). Of note, $8 \%$ of the psoriasis patients surveyed were taking a beta-blocker, and $12 \%$ an ACE inhibitor.

In patients taking multiple systemic therapies, drug interactions are another potential problem that must be considered when choosing the best option for the patient with numerous concomitant diseases. Of the available systemic
Table 1. Drugs as trigger factors for psoriasis.

\begin{tabular}{ll}
\hline Association & Drug Class \\
\hline Known & Beta-blockers \\
& Lithium \\
& Hydroxychloroquine \\
& Tetracycline \\
Likely & Angiotensin-converting enzyme (ACE) inhibitors \\
& Nonsteroidal antiinflammatory drugs (NSAID) \\
& Interferons \\
& Terbinafine \\
& Multiple \\
Case report & Efalizumab (which may result in transient \\
"New" & Tutrophilic dermatosis) \\
& in pustular transformation of plaque-type psoriasis) \\
\hline
\end{tabular}

antipsoriatic therapies, cyclosporin A and methotrexate bear a higher risk of drug interactions, whereas the biologics and fumaric acid esters have less (Table 2). All systemic medications for the treatment of psoriasis as well as existing 
Table 2. The risk of drug interactions.

\begin{tabular}{ll}
\hline Risk & Drug \\
\hline High & Cyclosporin A \\
& Methotrexate \\
Low & Leflunomide \\
& Retinoids \\
None & Fumaric acid esters \\
& Biologics \\
\hline
\end{tabular}

comorbidities must be reviewed, and drugs potentially triggering psoriasis should be avoided if possible.

The European League Against Rheumatism (EULAR) recently published recommendations for cardiovascular risk management in patients with RA and other forms of inflammatory arthritis including PsA ${ }^{36}$. EULAR recommends annual cardiovascular risk assessment using national guidelines for all patients with PsA. Any cardiovascular risk factors identified should be managed according to local guidelines; however, if local guidelines are not available, cardiovascular risk management should follow the Systematic Coronary Risk Evaluation (SCORE) $\operatorname{model}^{37}$, which is similar to the Framingham risk score. In addition to appropriate cardiovascular risk management, aggressive suppression of the inflammatory process was recommended to further lower the cardiovascular risk.

Comprehensive monitoring. Managing the comorbidities associated with psoriasis and PsA should not be limited to patients' skin and joint symptoms. Dermatologists in private practice can detect developing comorbidities early. According to a recent consensus under the guidance of the National Psoriasis Foundation, a comprehensive investigation of psoriasis patients should include measurement every 2 years of blood pressure, pulse, and body mass index (BMI); measurement every 5 years [every 2 years in patients with additional risk factors (e.g., positive family history, diabetes mellitus, smoking)] of fasting blood glucose and lipids; and assessment of joint status with validated screening questionnaires at every visit ${ }^{32,38}$. Validated screening questionnaires such as ToPAS (Toronto Psoriatic Arthritis Screening) or PASE (Psoriatic Arthritis Screening and Evaluation) should also be utilized to observe signs of developing joint involvement ${ }^{32}$. These simple observations will promote early detection and targeted transferral when necessary, protecting patients from avoidable health problems.

Future research agenda. It is well understood that psoriasis and PsA are associated with comorbidities that have a substantial influence on patients' well-being. Future research will need to clarify the pathogenetic links between psoriasis/PsA and its comorbidities. As there is evidence for the former driving the latter, the comorbidities may simply be complications of undertreated psoriasis and PsA. Consequently, the hypothesis that continuous systemic antipsoriat- ic therapy may reduce cardiovascular risk should be addressed in prospective clinical trials. A single-center open-label pilot trial is under way, and preliminary observations from it are encouraging; a multicenter followup study will be required to validate the results.

Recent publications have identified some biomarkers that identify psoriasis as a chronic systemic inflammatory disorder ${ }^{31,39}$. Thus, clinical assessment of the skin and joints in psoriasis and PsA, such as with the PASI, may need to be complemented with laboratory measures. Future projects should validate potential biomarkers for the systemic inflammatory burden of patients with psoriasis and PsA.

\section{REFERENCES}

1. Schön MP, Boehncke WH. Psoriasis. N Engl J Med 2005;352:1899-912.

2. Nestle FO, Kaplan DH, Barker J. Psoriasis. N Engl J Med 2009;361:496-509

3. Fitzgerald O, Winchester R. Psoriatic arthritis: from pathogenesis to therapy. Arthritis Res Ther 2009;11:214.

4. McGonagle D, Benjamin M, Tan AL. The pathogenesis of psoriatic arthritis and associated nail disease: not autoimmune after all? Curr Opin Rheumatol 2009;21:340-7.

5. McGonagle D, McDermott MF. A proposed classification of the immunological diseases. PLoS Med 2006;3:e297.

6. Boehncke WH. Immunomodulatory drugs for psoriasis. BMJ 2003;327:634-5.

7. Menter A, Griffiths CE. Current and future management of psoriasis. Lancet 2007;370:272-84.

8. Maradit-Kremers H, Nicola PJ, Crowson CS, Ballman KV, Gabriel SE. Cardiovascular death in rheumatoid arthritis: a population-based study. Arthritis Rheum 2005;52:722-32.

9. Popa C, Netea MG, Radstake T, van der Meer JW, Stalenhoef AF, van Riel PL, et al. Influence of anti-tumour necrosis factor therapy on cardiovascular risk factors in patients with active rheumatoid arthritis. Ann Rheum Dis 2005;64:303-5.

10. Mattey DL, Glossop JR, Nixon NB, Dawes PT. Circulating levels of tumor necrosis factor receptors are highly predictive of mortality in patients with rheumatoid arthritis. Arthritis Rheum 2007;56:3940-8.

11. Dixon WG, Watson KD, Lunt M, Hyrich KL, Silman AJ, Symmons DP. Reduction in the incidence of myocardial infarction in patients with rheumatoid arthritis who respond to anti-tumor necrosis factor alpha therapy: results from the British Society for Rheumatology Biologics Register. Arthritis Rheum 2007;56:2905-12.

12. Henseler T, Christophers E. Disease concomitance in psoriasis. J Am Acad Dermatol 1995;32:982-6.

13. Gelfand JM, Troxel AB, Lewis JD, Kurd SK, Shin DB, Wang X, et al. The risk of mortality in patients with psoriasis: results from a population-based study. Arch Dermatol 2007;143:1493-9.

14. Mallbris L, Akre O, Granath F, Yin L, Lindelof B, Ekbom A, et al. Increased risk for cardiovascular mortality in psoriasis inpatients but not in outpatients. Eur J Epidemiol 2004;19:225-30.

15. Gelfand JM, Neimann AL, Shin DB, Wang X, Margolis DJ, Troxel AB. Risk of myocardial infarction in patients with psoriasis. JAMA 2006;296:1735-41.

16. Ludwig RJ, Herzog C, Rostock A, Ochsendorf FR, Zollner TM, Thaci D, et al. Psoriasis: a possible risk factor for development of coronary artery calcification. Br J Dermatol 2007;156:271-6.

17. Wong K, Gladman DD, Husted J, Long JA, Farewell VT. Mortality studies in psoriatic arthritis: results from a single outpatient clinic. I. Causes and risk of death. Arthritis Rheum 1997;40:1868-72. 
18. Ali Y, Tom BD, Schentag CT, Farewell VT, Gladman DD. Improved survival in psoriatic arthritis with calendar time. Arthritis Rheum 2007;56:2708-14.

19. Gladman DD, Ang M, Su L, Tom BD, Schentag CT, Farewell VT. Cardiovascular morbidity in psoriatic arthritis. Ann Rheum Dis 2009;68:1131-5.

20. Bruce IN, Schentag CT, Gladman DD. Hyperuricemia in psoriatic arthritis: prevalence and associated features. J Clin Rheumatol 2000;6:6-9.

21. Gonzalez-Gay MA, Gonzalez-Juanatey C, Vazquez-Rodriguez TR, Gomez-Acebo I, Miranda-Filloy JA, Paz-Carreira J, et al. Asymptomatic hyperuricemia and serum uric acid concentration correlate with subclinical atherosclerosis in psoriatic arthritis patients without clinically evident cardiovascular disease. Semin Arthritis Rheum 2009;39:157-62.

22. Baker JF, Krishnan E, Chen L, Schumacher HR. Serum uric acid and cardiovascular disease: recent developments, and where do they leave us? Am J Med 2005; 118:816-26.

23. Tam LS, Shang Q, Li EK, Tomlinson B, Chu TT, Li M, et al. Subclinical carotid atherosclerosis in patients with psoriatic arthritis. Arthritis Rheum 2008;59:1322-31.

24. Eder L, Zisman D, Barzilai M, Laor A, Rahat M, Rozenbaum M, et al. Subclinical atherosclerosis in psoriatic arthritis: a case-control study. J Rheumatol 2008;35:877-82.

25. Gonzalez-Juanatey C, Llorca J, Amigo-Diaz E, Dierssen T, Martin J, Gonzalez-Gay MA. High prevalence of subclinical atherosclerosis in psoriatic arthritis patients without clinically evident cardiovascular disease or classic atherosclerosis risk factors. Arthritis Rheum 2007;57:1074-80.

26. Strand V. Are COX-2 inhibitors preferable to non-selective non-steroidal anti-inflammatory drugs in patients with risk of cardiovascular events taking low-dose aspirin? Lancet 2007;370:2138-51.

27. Sattar N, Crompton P, Cherry L, Kane D, Lowe G, McInnes IB. Effects of tumor necrosis factor blockade on cardiovascular risk factors in psoriatic arthritis: a double-blind, placebo-controlled study. Arthritis Rheum 2007;56:831-9.

28. Tam L, Li EK, Shang Q, Tomlinson B, Chu TT, Li M, et al. TNF-alpha blockade is associated with reduction of carotid intima-media thickness for patients with active psoriatic arthritis - a pilot study. Ann Rheum Dis 2009;68 Suppl 3:659.
29. Angel K, Provan SA, Gulseth HL, Mowinckel P, Kvien TK, Atar D. Tumor necrosis factor-alpha antagonists improve aortic stiffness in patients with inflammatory arthropathies: a controlled study. Hypertension 2009;55:333-8.

30. Boehncke WH, Buerger C, Boehncke S. Co-morbidities in psoriasis vulgaris. Hautarzt 2009;60:116-21.

31. Boehncke S, Thaci D, Beschmann H, Ludwig RJ, Ackermann H, Badenhoop K, et al. Psoriasis patients show signs of insulin resistance. Br J Dermatol 2007;157:1249-51.

32. Boehncke WH, Boehncke S, Schon MP. Managing comorbid disease in patients with psoriasis. BMJ 2010;340:b5666.

33. Naldi L, Chatenoud L, Linder D, Belloni Fortina A, Peserico A, Virgili AR, et al. Cigarette smoking, body mass index, and stressful life events as risk factors for psoriasis: results from an Italian case-control study. J Invest Dermatol 2005;125:61-7.

34. Nast A, Kopp I, Augustin M, Banditt KB, Boehncke WH, Follmann $\mathrm{M}$, et al. German evidence-based guidelines for the treatment of Psoriasis vulgaris (short version). Arch Dermatol Res 2007;299:111-38.

35. Gerdes S, Zahl VA, Knopf H, Weichenthal M, Mrowietz U. Comedication related to comorbidities: a study in 1203 hospitalized patients with severe psoriasis. Br J Dermatol 2008;159:1116-23.

36. Peters MJ, Symmons DP, McCarey D, Dijkmans BA, Nicola P, Kvien TK, et al. EULAR evidence-based recommendations for cardiovascular risk management in patients with rheumatoid arthritis and other forms of inflammatory arthritis. Ann Rheum Dis 2010;69:325-31.

37. Conroy RM, Pyorala K, Fitzgerald AP, Sans S, Menotti A, De Backer G, et al. Estimation of ten-year risk of fatal cardiovascular disease in Europe: the SCORE project. Eur Heart J 2003;24:987-1003

38. Kimball AB, Gladman D, Gelfand JM, Gordon K, Horn EJ, Korman NJ, et al. National Psoriasis Foundation clinical consensus on psoriasis comorbidities and recommendations for screening. J Am Acad Dermatol 2008;58:1031-42.

39. Tamagawa-Mineoka R, Katoh N, Kishimoto S. Platelet activation in patients with psoriasis: increased plasma levels of platelet-derived microparticles and soluble P-selectin. J Am Acad Dermatol 2010;62:621-6. 